\title{
Effects of medium chain triglycerides with organic acids on growth performance, fecal score, blood profiles, intestinal morphology, and nutrient digestibility in weaning pigs
}

\author{
Tae Wook Goh ${ }^{1}$, Jinsu Hong ${ }^{1,2}$, Dong Hyun You', Yeong Geol Han', \\ Seung Ok Nam ${ }^{1}$, and Yoo Yong Kim ${ }^{1, \star *}$
}

\author{
* Corresponding Author: Yoo Yong Kim \\ Tel: +82-2-878-5838, Fax: +82-2-878-5839 \\ E-mail: yooykim@snu.ac.kr \\ ${ }^{1}$ Department of Agricultural Biotechnology, \\ and Research Institute of Agriculture and \\ Life Sciences, Seoul National University, \\ Seoul 08826, Korea \\ 2 Department of Animal Science, South \\ Dakota State University, Brookings, SD \\ 57006, USA \\ ORCID \\ Tae Wook Goh \\ https://orcid.org/0000-0003-4705-3348 \\ Jinsu Hong \\ https://orcid.org/0000-0002-3401-3024 \\ Dong Hyun You \\ https://orcid.org/0000-0002-5946-9466 \\ Yeong Geol Han \\ https://orcid org/0000-0001-7869-1938 \\ Seung Ok Nam \\ https://orcid.org/0000-0002-9869-1895 \\ Yoo Yong Kim \\ https://orcid.org/0000-0001-8121-3291
}

Submitted Oct 13, 2021; Revised Dec 14, 2021; Accepted Jan 29, 2022
Objective: This study was conducted to evaluate the effects of medium chain triglycerides (MCT) with organic acids (OA) on growth performance, fecal score, blood profiles, intestinal morphology, and nutrient digestibility in weaning pigs.

Methods: A total of 120 weaning pigs ([Yorkshire $\times$ Landrace $] \times$ Duroc) with an average body weight (BW) of $8.00 \pm 0.87 \mathrm{~kg}$ were assigned in five treatments considering sex and initial BW in 3 replications with 8 pigs per pen in a randomized complete block design. The experimental diets included a corn-soybean meal based basal diet with or without $0.1 \%$ or $0.2 \%$ MCT and $0.1 \%$ OA. The pigs were fed the diets for 5 weeks (phase 1, 0 to 2 weeks; phase 2, 3 to 5 weeks). A total of 15 barrows with an average $B W$ of $12.48 \pm 0.37 \mathrm{~kg}$ were used to evaluate the nutrient digestibility by total collection method. The BW and feed intake were measured at the end of each phase. Blood samples and small intestine samples were collected at the end of each phase, too.

Results: Supplementing 0.1\% MCT with $0.1 \%$ OA showed greater BW for week 5 and average daily gain $(A D G)$ for overall period than control diet. Supplementing $0.1 \%$ MCT increased $(\mathrm{p}<0.05)$ ADG and improved $(\mathrm{p}<0.05)$ gain:feed ratio for phase 1. Dietary MCT and OA did not affect the fecal score and blood concentration of cortisol, immunoglobulin G, tumor necrosis factor- $\alpha$, interleukin- $1 \beta$ (IL-1 $\beta$ ), IL- 6 , and IL-10 in weaning pigs. Pigs fed the diets with $0.1 \%$ MCT had greater $(\mathrm{p}<0.05)$ villus height of duodenum and ileum for phase 1 . Also, pigs fed the diet with $0.1 \%$ OA showed greater $(\mathrm{p}<0.05)$ villus height and villus height to crypt depth ratio of duodenum for phase 2 . There was no significant difference in nutrient digestibility and nitrogen retention of pigs.

Conclusion: Addition of $0.1 \%$ MCT with $0.1 \%$ OA in weaning pig's diet improved growth performance partly by enhancing intestinal morphology in weaning pigs.

Keywords: Growth Performance; Intestinal Morphology; Medium Chain Triglycerides; Organic Acid; Weaning Pig

\section{INTRODUCTION}

Weaning pigs experience highly stressful events when they are weaned from the sow [1]. To alleviate the detrimental effects of weaning, antibiotic growth promoters (AGPs) have been widely used in weaning pigs' diet. However, the use of AGPs in animal feed have been forbidden due to the antibiotic resistance problem [2]. Several feed additives as alternatives to AGPs have been proposed to overcome the increased mortality due to the ban on antibiotic use in animal feed [2]. Among the alternatives, medium chain triglycerides (MCT) and organic acids (OA) have been widely researched and applied in weanling pig diets as promising alternatives to AGPs [3]. 
Medium chain triglycerides have nutritional and metabolic effects including speeding up digestion and improving passive absorption and effects on oxidation status. Therefore, they are particularly useful for the improving nutritional ingestion in piglets [4]. It has been reported in many previous studies that MCT has a positive effect on improving the growth performance of piglets, especially during the second weeks after weaning [5]. In addition, MCT has antimicrobial effects on gram-positive cocci [6] and Escherichia coli (E. coli) [7] and protective effects on the villi [5].

Organic acids can lower gastric $\mathrm{pH}$ which is essential for the proper digestion of nutrients and in particular protein digestion for improving growth performance and prevention of infection by diarrhea-causing micro-organisms [8]. Organic acid and their salts, especially formic acid, and potassium formate, have the advantage of improving the feed intake and growth rate of piglets [8]. They also affect the gastrointestinal environment and the digestive process [9]. Moreover, they have effects on intracellular acidification, and possibly act to inhibit bacteria [9].

Although both MCT and OA have beneficial effects for weaning pigs, there are few studies on supplementing MCT and $\mathrm{OA}$ in weaning pigs' diet at the same time. Kuang et al [3] investigated the effects of combinations of MCT and OA growth performance in weaning pigs. Animals supplemented with $0.3 \%$ MCT and OA showed higher feed intake $(\mathrm{p}<0.01)$ during the first two experimental weeks and had a higher body weight $(\mathrm{BW})(\mathrm{p}<0.05)$ at the end of each of the four experimental weeks than the control. Lei et al [10] also conducted the experiment to compare the effects of MCT and $\mathrm{OA}$ on growth performance in weaning pigs. Weaning pigs fed the combination of $0.2 \%$ and $0.4 \%$ MCT and OA had higher $(\mathrm{p}<0.05)$ BW than those of control groups on day 7 , 14 , and 21 of the experiment.

However, there is insufficient evidence to verify the synergic effect of MCT and OA on growth performance, gastrointestinal environment, and nutrient absorption in weaning pigs. Thus, it was hypothesized that synergic effects of MCT and OA could improve gastrointestinal environment and nutrient absorption, leading to an improvement of growth performance in weaning pigs. Therefore, this study was conducted to evaluate the effects of MCT with OA on growth performance, fecal score, blood profiles, intestinal morphology, and nutrient digestibility in weaning pigs.

\section{MATERIALS AND METHODS}

All experimental procedures involving animals were conducted in accordance with the Animal Experimental Guidelines provided by the Seoul National University Institutional Animal Care and Use Committee (SNUIACUC; SNU-170424-3-1).

\section{Experimental animals and management} A total of 120 weaning pigs ([Yorkshire $\times$ Landrace $] \times$ Duroc) with initial BW of $8.00 \pm 0.87 \mathrm{~kg}$ were allotted to one of five treatments considering sex and initial BW in 3 replicates with 8 pigs per pen in a randomized complete block design.

Pigs were randomly allotted to their respective treatments by the experimental animal allotment program (EAAP) [11]. Pigs were housed in an environmentally controlled facility. The pens were fully concrete floored $(1.54 \times 1.96 \mathrm{~m})$ and equipped with a feeder and, water nipple. The experimental period was 5 weeks (phase 1, 0 to 2 weeks; phase 2, 3 to 5 weeks).

\section{Experimental design and diet}

Dietary treatments included: i) CON (corn-soybean meal (SBM) based diet), ii) LM (corn-SBM based diet $+0.1 \% \mathrm{MCT}$ ), iii) LMO (corn-SBM based diet + 0.1\% MCT + 0.1\% OA), iv) HM (corn-SBM based diet + $0.2 \% \mathrm{MCT}$ ), v) HMO (corn$\mathrm{SBM}$ based diet $+0.2 \% \mathrm{MCT}+0.1 \% \mathrm{OA}$ ). In the present study, the MCT and OA products were provided by E\&T company (E\&T CO., Ltd, Daejeon, Korea). The MCT product contained $49.65 \%$ C6:0 and $43.01 \%$ C8:0 and the OA product contained $65 \%$ calcium formate, $15 \%$ citric acid, $10 \%$ fumaric acid. Experimental diets were formulated for the 2 phases, including weaning phase 1 and weaning phase 2. All nutrients in the experimental diets except crude protein (CP) and metabolizable energy (ME) met or exceeded the nutrient requirements of the National Research Council (NRC) [12]. The ME and CP were determined to meet NRC [13]. The formula and chemical composition of the experimental diet are presented in Table 1 and 2.

\section{Growth performance}

Body weight and feed intake were measured at the end of each phase to calculate the average daily gain (ADG), average daily feed intake (ADFI), and gain:feed ratio (G:F ratio). In addition, feed given to all piglets was recorded each day, and feed waste in the feeder was recorded at the end of each phase.

\section{Fecal score}

Observations of fecal score were made every 08:00 throughout the feeding trial ( 35 days). Data were recorded by one trained researcher for each pen. Fecal scores were given according to the condition of feces $(0=$ normal feces; $1=$ moist feces; 2 = mild feces; 3 = watery diarrhea) [14]. The score of 2 or 3 was considered severe diarrhea. Slightly wet feces on the rump area were used to designate contaminated piglets. After recording data, we cleaned away the feces by wiping off the fecal areas or the pig's butt preparing for a new measurement the next day. 
Table 1. Formula and chemical compositions of the experimental diets during the weaning phase 1 (0 to 2 wk)

\begin{tabular}{|c|c|c|c|c|c|}
\hline \multirow{2}{*}{ Items } & \multicolumn{5}{|c|}{ Treatment $^{1)}$} \\
\hline & CON & LM & LMO & HM & HMO \\
\hline \multicolumn{6}{|l|}{ Ingredient (\%) } \\
\hline Soybean meal & 37.02 & 37.04 & 37.08 & 37.06 & 37.09 \\
\hline Soy oil & 1.84 & 1.81 & 1.88 & 1.79 & 1.86 \\
\hline Sweet whey powder & 4.00 & 4.00 & 4.00 & 4.00 & 4.00 \\
\hline DL-methionine 80\% & 0.05 & 0.04 & 0.04 & 0.04 & 0.04 \\
\hline L-threonine, 99\% & 0.04 & 0.04 & 0.03 & 0.03 & 0.04 \\
\hline MDCP & 1.43 & 1.43 & 1.43 & 1.43 & 1.43 \\
\hline Limestone & 1.06 & 1.06 & 1.06 & 1.06 & 1.06 \\
\hline Vitamin mix $^{2)}$ & 0.10 & 0.10 & 0.10 & 0.10 & 0.10 \\
\hline Organic acids ${ }^{4)}$ & 0.00 & 0.00 & 0.10 & 0.00 & 0.10 \\
\hline Total & 100.00 & 100.00 & 100.00 & 100.00 & 100.00 \\
\hline \multicolumn{6}{|c|}{ Chemical composition (calculated value) } \\
\hline $\mathrm{ME}(\mathrm{kcal} / \mathrm{kg})$ & $3,300.00$ & $3,300.01$ & $3,300.03$ & $3,300.01$ & $3,300.03$ \\
\hline SID lysine (\%) & 1.35 & 1.35 & 1.35 & 1.35 & 1.35 \\
\hline SID methionine (\%) & 0.35 & 0.35 & 0.35 & 0.35 & 0.35 \\
\hline SID threonine (\%) & 0.86 & 0.86 & 0.86 & 0.86 & 0.86 \\
\hline Calcium (\%) & 0.80 & 0.80 & 0.80 & 0.80 & 0.80 \\
\hline Total phosphorus (\%) & 0.65 & 0.65 & 0.65 & 0.65 & 0.65 \\
\hline \multicolumn{6}{|c|}{ Chemical composition (analyzed value) } \\
\hline Moisture (\%) & 8.90 & 8.76 & 8.93 & 8.80 & 8.95 \\
\hline
\end{tabular}

MDCP, mono-dicalcium phosphate; MCT, medium chain triglyceride; ME, metabolizable energy; SID, standard ileal digestibility; SBM, soybean meal, OA, organic acids.

1) CON, corn-SBM-based diet; LM, corn-SBM-based diet + MCT 0.1\%; HM, corn-SBM-based diet + MCT 0.2\%; LMO, corn-SBM-based diet + MCT 0.1\% + organic acids $0.1 \%$; HMO, corn-SBM-based diet + MCT 0.2\% + organic acids $0.1 \%$.

2) Following quantities of vitamins provided per kg of complete diet: vitamin $\mathrm{A}, 8,000 \mathrm{IU}$; vitamin $\mathrm{D}_{3}, 1,800 \mathrm{IU}$; vitamin $\mathrm{E}, 80 \mathrm{IU}$; vitamin $\mathrm{K}_{3}, 2$ mg; riboflavin, 7

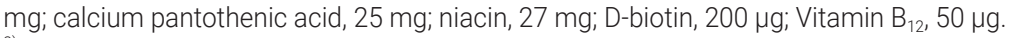

3) Following quantities of minerals provided per kg of complete diet: Fe, 150 mg; Cu, 105 mg; Mn, 51 mg; l, 1 mg; Se, 0.3 mg; Zn, 72 mg.

4) MCT and OA products were provided by E\&T company (E\&T CO., Ltd, Daejeon, Korea).

\section{Blood collection and analysis}

Blood samples were taken from the jugular vein of five pigs near the average BW in each treatment after 3 hours of fasting at initial day and the end of each phase for measuring cortisol, immunoglobulin $\mathrm{G}(\operatorname{IgG})$, tumor necrosis factor- $\alpha$ (TNF- $\alpha)$, interleukin-1 $\beta$ (IL-1 $\beta$ ), IL-6, and IL-10. All blood samples were collected in the serum tubes (SST II Advance; BD Vacutainer, Becton Dickinson, Plymouth, UK) and centrifuged at $1,957 \times \mathrm{g}$ and $4^{\circ} \mathrm{C}$ for $15 \mathrm{~min}$ (5810R; Eppendorf, Hamburg, centrifuge $5810 \mathrm{R}$, Germany). The sera were carefully transferred to $1.5 \mathrm{~mL}$ microtubes (MCT-150-C; AXYGEN. INC, Glendale, AZ, USA) and stored at $-20^{\circ} \mathrm{C}$ until further analysis. The enzyme-linked immunosorbent assay (ELISA) were used to measure the following parameters: cortisol concen- tration (Microplate Reader, VERSA Max, Swine Cortisol ELISA Kit; Molecular Devices, San Jose, CA, USA) to assess stress; IgG concentration (Microplate Reader, VERSA Max, Pig IgG ELISA Kit; Molecular Devices, USA) to determine immune status; TNF- $\alpha$ (Microplate Reader, VERSA Max, Quantikine Porcine TNF-a TNFSF2 Immunoassay; Molecular Devices, USA), IL-1 $\beta$ (Microplate Reader, VERSA Max, Quantikine Porcine IL-1 $\beta$ IL-1F2 Immunoassay; Molecular Devices, USA), and IL-6 (Microplate Reader, VERSA Max, Porcine IL-6 Immunoassay; Molecular Devices, USA) concentrations to assess the degree of inflammatory cytokine changes; and IL-10 concentration (Microplate Reader, VERSA Max, Quantikine Porcine IL-10 Immunoassay; Molecular Devices, USA) to investigate the degree of ant-inflammatory 
Table 2. Formula and chemical compositions of the experimental diets during the weaning phase 2 ( 3 to 5 wk)

\begin{tabular}{|c|c|c|c|c|c|}
\hline \multirow{2}{*}{ Items } & \multicolumn{5}{|c|}{ Treatment $^{1)}$} \\
\hline & CON & LM & LMO & HM & HMO \\
\hline \multicolumn{6}{|l|}{ Ingredient (\%) } \\
\hline Soybean meal & 32.29 & 32.31 & 32.33 & 32.32 & 32.35 \\
\hline Soy oil & 1.78 & 1.75 & 1.83 & 1.73 & 1.80 \\
\hline Sweet whey powder & 2.00 & 2.00 & 2.00 & 2.00 & 2.00 \\
\hline DL-methionine 80\% & 0.01 & 0.01 & 0.01 & 0.01 & 0.01 \\
\hline L-threonine, 99\% & 0.00 & 0.00 & 0.00 & 0.00 & 0.00 \\
\hline MDCP & 1.22 & 1.22 & 1.22 & 1.22 & 1.22 \\
\hline Limestone & 0.95 & 0.95 & 0.95 & 0.95 & 0.95 \\
\hline Vitamin $\mathrm{mix}^{2}$ ) & 0.10 & 0.10 & 0.10 & 0.10 & 0.10 \\
\hline Organic acids ${ }^{4)}$ & 0.00 & 0.00 & 0.10 & 0.00 & 0.10 \\
\hline Total & 100.00 & 100.00 & 100.00 & 100.00 & 100.00 \\
\hline \multicolumn{6}{|c|}{ Chemical composition (calculated value) } \\
\hline $\mathrm{ME}(\mathrm{kcal} / \mathrm{kg})$ & $3,300.01$ & $3,300.00$ & $3,300.01$ & $3,300.04$ & $3,300.02$ \\
\hline SID lysine (\%) & 1.15 & 1.15 & 1.15 & 1.15 & 1.15 \\
\hline SID methionine (\%) & 0.30 & 0.30 & 0.30 & 0.30 & 0.30 \\
\hline SID threonine (\%) & 0.74 & 0.74 & 0.74 & 0.74 & 0.74 \\
\hline Calcium (\%) & 0.70 & 0.70 & 0.70 & 0.70 & 0.70 \\
\hline Total phosphorus (\%) & 0.60 & 0.60 & 0.60 & 0.60 & 0.60 \\
\hline \multicolumn{6}{|c|}{ Chemical composition (analyzed value) } \\
\hline Moisture (\%) & 9.02 & 9.24 & 9.33 & 9.22 & 8.78 \\
\hline
\end{tabular}

MDCP, mono-dicalcium phosphate; MCT, medium chain triglyceride; ME, metabolizable energy; SID, standard ileal digestibility; SBM, soybean meal; OA, organic acids.

1) CON, corn-SBM-based diet; LM, corn-SBM-based diet + MCT 0.1\%; HM, corn-SBM-based diet + MCT 0.2\%; LMO, corn-SBM-based diet + MCT 0.1\% + organic acids $0.1 \%$; HMO, corn-SBM-based diet + MCT $0.2 \%$ + organic acids $0.1 \%$.

2) Following quantities of vitamins provided per kg of complete diet: Vitamin $A, 8,000$ IU; Vitamin $\mathrm{D}_{3}, 1,800 \mathrm{IU}$; Vitamin E, 80 IU; Vitamin $\mathrm{K}_{3}, 2$ mg; riboflavin, 7 mg; calcium pantothenic acid, 25 mg; niacin, 27 mg; D-biotin, $200 \mu g$; Vitamin B12, $50 \mu \mathrm{g}$.

3) Following quantities of minerals provided per kg of complete diet: Fe, 150 mg; Cu, 105 mg; Mn, 51 mg; I, 1 mg; Se, 0.3 mg; Zn, 72 mg.

4) $\mathrm{MCT}$ and OA products were provided by E\&T company (E\&T CO., Ltd, Daejeon, Korea).

cytokine changes.

\section{Nutrient digestibility}

A total of 15 crossbred barrows, averaging $12.48 \pm 0.37 \mathrm{~kg}$ BW were allotted to individual metabolic crates $(40 \times 80 \times 90$ $\mathrm{cm}$ ) in a completely randomized design with 3 replicates to evaluate the nutrient digestibility and nitrogen retention. The total collection method was used to determine the apparent total tract digestibility of dry matter (DM), CP, crude ash, and crude fat [15]. After a 5-day adaptation period, there was a 5-day collection period. To determine the first and last day of collection, $8 \mathrm{~g}$ of ferric oxide and chromium oxide were added to the first and last experimental diets as selection markers. During the experimental period, all pigs were fed the phase 2 diets twice a day, which provided three times the maintenance energy [16], at 7:00 and 19:00, and water was provided ad libitum. Collection of feces was started when the ferric oxide appeared in the feces and kept until the appearance of chromium oxide in the feces. Urine samples were collected during collection period in plastic containers containing $50 \mathrm{~mL}$ of $4 \mathrm{~N} \mathrm{H}_{2} \mathrm{SO}_{4}$ to prevent evaporation of nitrogen prior to nitrogen retention analysis. Fecal and urinary samples were stored at $-20^{\circ} \mathrm{C}$ until the end of collection period and the feces were dried in a drying oven at $60^{\circ} \mathrm{C}$ for $72 \mathrm{~h}$ and then ground to $1 \mathrm{~mm}$ in a Wiley mill (CT 193 Cyclotec; FOSS, Höganäs, Sweden) for chemical analysis including moisture, $\mathrm{CP}$, crude fat, and crude ash contents by the Association of Official Analytical Chemists (AOAC) methods [17]. 


\section{Intestinal morphology}

Three piglets of near average BW were selected for each treatment and euthanized at the end of each phase to collect small intestinal tissues. After removal of the digestive system, 6 to $8 \mathrm{~cm}$ samples from the middle of each duodenum, jejunum, and ileum were cut out, cleaned, and stored in $10 \%$ neutralbuffered formalin solution until further morphological analysis. The samples were then cut into two parts for each segment for measurement of the cross section and length of the intestine surface and then processed by the standard paraffin method. Sections $(2$ to $3 \mathrm{~cm}$ ) were stained with hematoxylin and eosin, and examined under a light microscope (Leica DM500 microscope with Leica DFC425; Leica Microsystems Inc., Morrisville, NC, USA) was used for image analysis. The villus height $(\mathrm{VH})$ was measured from the tip to the villus crypt junction. The crypt depth (CD) was measured as the depth between adjacent villi.

\section{Chemical analysis}

The diets and feces were ground by Wiley mill (CT 193 Cyclotec; FOSS, Sweden) and then analyzed for DM (procedure 967.03; [17]), ash (procedure 923.03; [17]), ether extract (procedure 920.39; [17]). The nitrogen content was analyzed by using the Kjeldahl procedure with Kjeltec (KjeltecTM 2200; Foss Tecator, Sweden) and calculating the CP content (nitrogen $\times 6.25$; procedure 981.10 ; [17]).

\section{Statistical analyses}

Differences among all treatments were carried out by com- paring means according to the least significant difference multiple range tests, using the general linear model protocol of SAS (SAS Inst. Inc., Cary, NC, USA). Each pen was used as the experimental unit for growth performance and fecal score, while individual pig was used as the experimental unit for blood profiles, intestinal morphology, and nutrient digestibility. The data were analyzed as a $2 \times 2$ factorial treatment arrangements to find out the main effects of MCT and OA, as well as interaction between MCT and OA. Also, the data were analyzed by analysis of variance to compare the effects of MCT and OA against control. Differences were declared significant at $\mathrm{p}<0.05$, while $0.05 \leq \mathrm{p}<0.10$ was considered to indicate a trend in the data.

\section{RESULTS AND DISCUSSION}

\section{Growth performance}

The dietary effects of MCT with OA on growth performance are presented in Table 3. An interaction between dietary MCT and OA was observed in the ADG for 3 to 5 weeks (MCTx $\mathrm{OA}, \mathrm{p}=0.04$ ) such that there was no $0.1 \%$ OA effect with $0.1 \%$ MCT level, whereas addition of $0.1 \%$ OA with $0.2 \%$ MCT level decreased the ADG of weaning pigs. Treatment LMO showed greater BW for 5 week and ADG for 3 to 5 weeks and overall period than CON treatment. Supplementing $0.2 \%$ MCT decreased ADG and G:F ratio for 0 to 2 weeks (MCT, $\mathrm{p}=0.04, \mathrm{p}=0.01$ ). Furthermore, $0.2 \% \mathrm{MCT}$ decreased $\mathrm{ADG}$ of pigs for 3 to 5 weeks and overall period (MCT, $\mathrm{p}=$ $0.01, \mathrm{p}=0.01)$.

Table 3. Effects of medium chain triglycerides and organic acids on growth performance in weaning pigs ${ }^{1)}$

\begin{tabular}{|c|c|c|c|c|c|c|c|c|c|c|}
\hline \multirow{2}{*}{ Items } & \multicolumn{5}{|c|}{ Treatment $^{2)}$} & \multirow{2}{*}{ SEM } & \multicolumn{4}{|c|}{$\mathrm{p}$-value } \\
\hline & CON & LM & LMO & HM & HMO & & Diet & MCT & OA & MCT $\times O A$ \\
\hline \multicolumn{11}{|c|}{ Body weight (kg) } \\
\hline $2 w k$ & 9.60 & 10.07 & 10.46 & 9.58 & 9.47 & 0.265 & 0.14 & 0.30 & 0.84 & 0.72 \\
\hline $5 w k$ & $18.12^{\mathrm{bc}}$ & $19.52^{\mathrm{ab}}$ & $20.10^{a}$ & $18.89^{\mathrm{abc}}$ & $17.75^{\mathrm{c}}$ & 0.382 & 0.03 & 0.09 & 0.73 & 0.31 \\
\hline \multicolumn{11}{|c|}{ Average daily gain (g) } \\
\hline 0 to 5 wk & $289^{\mathrm{bc}}$ & $329^{\mathrm{ab}}$ & $346^{\mathrm{a}}$ & $311^{\mathrm{abc}}$ & $279^{c}$ & 8.5 & 0.03 & 0.01 & 0.56 & 0.10 \\
\hline \multicolumn{11}{|c|}{ Average daily feed intake (g) } \\
\hline 0 to $2 w k$ & 231 & 239 & 258 & 228 & 211 & 9.9 & 0.53 & 0.27 & 0.95 & 0.49 \\
\hline 3 to 5 wk & 685 & 737 & 751 & 693 & 657 & 18.7 & 0.46 & 0.12 & 0.79 & 0.54 \\
\hline 0 to 5 wk & 444 & 476 & 487 & 448 & 423 & 12.3 & 0.44 & 0.13 & 0.81 & 0.52 \\
\hline
\end{tabular}

SEM, standard error of the mean; MCT, medium chain triglyceride; OA, organic acids; SBM, soybean meal.

1) A total 120 weaning pigs was fed from average initial body $8.00 \pm 0.694 \mathrm{~kg}$ and average final body weight was $18.87 \pm 2.160 \mathrm{~kg}$.

${ }^{2)}$ CON, corn-SBM-based diet; LM, corn-SBM-based diet + MCT 0.1\%; HM, corn-SBM-based diet + MCT 0.2\%; LMO, corn-SBM-based diet + MCT 0.1\% + organic acids $0.1 \%$; HMO, corn-SBM-based diet + MCT $0.2 \%+$ organic acids $0.1 \%$.

a-c Means in a same row with different superscript letters significantly differ $(p<0.05)$. 
In the previous study of Kuang et al [3], feed intake had been highly increased by supplementation of $0.3 \% \mathrm{MCT}$ and $\mathrm{OA}$ in 21-day-old weaning pigs for 1 week and 2 weeks. Additionally, BW, ADG, and the G:F ratio were increased by supplementation of MCT with OA during 0 to 1 week. The reason for the good growth performance in the treatment group given MCT with OA additions was due to the effects of MCT as well as the effects of OA in increasing the feed intake and growth rate.

Most previous studies have reported that the supplemental effect of MCT on the growth performance of weaning pigs was positive during phase 1 . Loh et al [18] reported that all treatment groups given $5 \mathrm{~mL}$ MCT had higher ADG compared to the control group during phase 1 [18]. Similarly, Dierick et al [19] observed that two treatments containing 2.5\% MCT showed the highest ADG during 0 to 14 days and had a more than $10 \%$ higher ADG and a $3 \%$ better feed conversion compared to the control.

The improvement of ADG and feed efficiency of weaning pigs fed the diet with MCT were due to the specific physiological and biological characteristics of MCT. Fatty acids are usually divided into short-chain triglycerides (SCT), MCT, and long-chain triglycerides (LCT) according to their hydrocarbon chain length. LCT comprise of fatty acid chains ranging between 13-22 carbons in length. Compared with LCT, MCT are smaller in molecular weight, water soluble, have lower smoke point and no essential fatty acids. Also, MCT digestion is rapid and simple. MCT do not stimulate cholecystokinin and pancreatic enzymes from the pancreas, and they are directly absorbed into portal circulation [20]. Several effects of these complex MCT would contribute to better absorption capacity of the intestinal tract and lead to a better growth performance [18]. Furthermore, unlike LCT, MCT do not require carnitine. In the intracellular space, LCT bind to carnitine and are transported to the mitochondria for subsequent $\beta$-oxidation while MCT do not rely on the carnitine acyltransferase system for transport into the mitochondria for $\beta$-oxidation. This provides MCT with a more rapid metabolism and improves their utilization even in protein-deficient conditions [21]. Therefore, MCT have the advantage that they can be used immediately as an energy source and give positive results when was added to weaning pig diets to meet their energy needs. On the other hands, high dosage of dietary MCT may have negative influence on the growth performance of weaning pigs. In earlier experiments by Allee et al [22], high doses (10\%) of MCT lowered piglet performance when compared to tallow, lard, or coconut oil. Also, supplementing 2.1\% MCT decreased ADFI of weaning pigs linearly between day 1 and 28 among treatments [23]. The treatment with $0.2 \%$ MCT showed lower growth performance than the treatment with $0.1 \% \mathrm{MCT}$ in this experiment. This might be due to not only fatty acid oxidation, which could increase satiety and reduce feed intake, but also the results of lower villous height. The intestinal morphology data in this experiment showed that the $\mathrm{VH}$ responsible for nutrient digestion and absorption was relatively shorter in the high MCT-added treatment than in the low-MCT treatment. However, it is difficult to say that adding a higher content of MCT had a negative effect on growth performance in this experiment. Further studies with higher contents of MCT would be warranted.

Consequently, when $0.2 \%$ MCT was added, the growth performance was decreased because of fatty acid oxidation which could increase satiety and reduce feed intake and relatively short villous height which could affect digestion and absorption of nutrients. However, supplementation with $0.1 \%$ MCT significantly improved ADG and feed efficiency. Furthermore, supplementing $0.1 \%$ MCT with $0.1 \%$ OA showed significantly higher results in growth performance compared to the control. Our ADG results for MCT and OA interaction effect cannot be related with intestinal morphology results because no significant difference was found in the MCT and OA interaction effect in intestinal morphology in our experiment. Therefore, additional studies related to combination of MCT and OA should be conducted to explain clearly about the interaction effects of MCT and OA to see the growth performance related to the intestinal morphology.

\section{Fecal score}

The effects of MCT with OA on the fecal score are presented in Table 4. No significant difference was observed during the whole experimental period.

Yen et al [14] have measured the fecal score and the number of diarrhea pigs with and without MCT supplementation ( $3 \% \mathrm{MCT})$, and the fecal scores and the number of diarrheal pigs in the MCT supplemented treatment group were lower than those in the control. Tee et al [24] also noted that diarrhea occurred less often in treatment groups given $2 \mathrm{~mL}$ MCT compared to the control. These findings could be supported by another previous study which showed that adding MCT in the feed controlled the antibacterial response of pigs. Petschow et al [25] reported that there were strong in vitro and in vivo antibacterial effects of medium chain fatty acids in the pig proximal small intestine without growth-promoting antibiotics. These results indicated that pathogenic bacteria, including Vibrio cholera, Salmonella typhimurium, Shigella sonnei, Haemophilus influenzae, and E. coli were inactivated by medium chain fatty acids or their monoglycerides [25]. Furthermore, MCT may reduce the proliferation of the pathogenic bacteria, which eventually could lead to more energy used for animal growth.

In addition, Tsiloyiannis et al [26] have reported on the effect of OA on pig diarrhea by comparing the effects of OA types, and diarrhea was less frequent in all treatment groups 
Table 4. Effects of medium chain triglycerides and organic acids on the fecal score in weaning pigs

\begin{tabular}{|c|c|c|c|c|c|c|c|c|c|c|}
\hline \multirow{2}{*}{ Items } & \multicolumn{5}{|c|}{ Treatment ${ }^{1)}$} & \multirow{2}{*}{ SEM } & \multicolumn{4}{|c|}{ p-value } \\
\hline & CON & LM & LMO & HM & HMO & & Diet & MCT & OA & MCT×OA \\
\hline \multicolumn{11}{|l|}{ Fecal score ${ }^{2)}$} \\
\hline 3 to $5 w k$ & 1.16 & 0.75 & 0.75 & 0.88 & 0.91 & 0.054 & 0.19 & 0.31 & 0.73 & 0.73 \\
\hline 0 to $5 \mathrm{wk}$ & 1.41 & 0.98 & 0.95 & 1.04 & 1.16 & 0.050 & 0.10 & 0.22 & 0.65 & 0.50 \\
\hline
\end{tabular}

SEM, standard error of the mean; MCT, medium chain triglyceride; OA, organic acids; SBM, soybean meal.

1) CON, corn-SBM based diet; LM, corn-SBM based diet + MCT 0.1\%; LMO, corn-SBM based diet + MCT 0.1\% + organic acids $0.1 \%$; HM, corn-SBM based diet + MCT 0.2\%; HMO, corn-SBM based diet + MCT $0.2 \%$ + organic acids $0.1 \%$.

2) Fecal score: 0 , normal feces; 1 , moist feces; 2 , mild diarrhea; 3 , watery diarrhea.

given OA than in the control without OA. This was because piglets had reduced intestinal $\mathrm{pH}$ after ingesting $\mathrm{OA}$, which inhibited the growth of bacteria, especially E. coli [26]. Also, all treatments including $\mathrm{OA}(0.2 \%$ unprotected $\mathrm{OA}, 0.1 \%$ protected OA, $0.2 \%$ protected $\mathrm{OA}$ ) had lower fecal score compared to the control in weaning pigs during whole experimental periods [27]. This experiment like Tsiloyiannis et al [26] showed that OA could prevent or reduce diarrhea in weaning pigs by inhibiting the growth of pathogenic bacteria in the gastrointestinal tract.

One of the reasons why the results were different with previous research was the MCT or OA content. Yen et al [14] added $3 \%$ MCT while our experiment was added $0.1 \%$ or $0.2 \%$ MCT. Also, $0.1 \%$ or $0.2 \%$ OA was supplemented while our experiment was supplemented $0 \%$ or $0.1 \%$ [27]. In conclusion, no significant difference in fecal score was found.

\section{Blood profiles}

The effects of MCT with OA on blood profiles of weaning pigs are presented in Table 5. As a result of the analysis, there was no statistically significant difference in blood profiles during the whole experimental period.

Cortisol is a commonly measured indicator of stress [28]. It was measured in the present study because Han et al [29] reported that MCT could reduce the level of cortisol, to lower levels that seen with antibiotics. However, no significant difference was observed in the current study.

The serum IgG, TNF- $\alpha$, IL- 6 , IL- $1 \beta$, and IL-10 can be considered as an indicator for measurement of immunological responses. No significant differences were found in the current study. However, Kuang et al [3] reported that weaning pigs supplemented with the combinations of $0.3 \% \mathrm{MCT}$ and OA had lower plasma TNF- $\alpha(\mathrm{p}<0.05)$ and higher plasma $\operatorname{IgG}(\mathrm{p}<0.05)$ concentrations than the weaning pigs fed with the control diets. The increased plasma IgG concentration suggested that the immunity of weaning pigs was improved by the combinations of MCT and OA intake. In addition, there was decreased plasma TNF- $\alpha$ concentration and downregulated TNF- $\alpha$ expression in the small intestine of the combination of MCT and OA fed weaning pigs in this study.
The decrease in feed intake and growth observed in diseased or immunologically challenged pigs was considered to be the result of increased activities of pro-inflammatory cytokines such as TNF- $\alpha$ and IL-1 [30].

The reasons why results of this current experiment were not the same as those of Kuang et al [3], are probably due to the difference in composition and content of the added MCT and OA. In the case of Kuang et al [3], 0.3\% MCT and OA were added. MCT was mainly composed with a mixture of lauric acid, myristic acid and capric acid and OA consisted of calcium formate, calcium lactate, citric acid, respectively. On the other hand, $0.1 \%$ or $0.2 \% \mathrm{MCT}$ and $0 \%$ and $0.1 \% \mathrm{OA}$ were added in this experiment. MCT mainly contained caproic acid and caprylic acid and OA mainly contained calcium formate, citric acid, fumaric acid.

The current study did not show any significant differences in blood immune parameters.

\section{Intestinal morphology}

The effects of MCT and OA on small intestinal morphology are presented in Table 6. Supplementing 0.1\% MCT increased $\mathrm{VH}$ in the duodenum (MCT, $\mathrm{p}=0.04)$ and ileum (MCT, $\mathrm{p}=$ 0.03 ) during phase 1 ( 0 to 2 weeks). In addition, supplementing $0.1 \% \mathrm{OA}$ increased duodenal $\mathrm{VH}(\mathrm{OA}, \mathrm{p}=0.01)$ and the $\mathrm{VH}: \mathrm{CD}$ ratio $(\mathrm{OA}, \mathrm{p}=0.04)$ during phase 2 ( 3 to 5 weeks).

Czernichow et al [31] have noted that supplementation with 50\% MCT + 50\% LCT could promote mucosal growth and enhance epithelial cell regeneration to create better-developed intestinal tracts in adult rats compared to $100 \%$ LCT treatments. Loh et al [18] have reported that compared with the control, supplementing $5 \mathrm{~mL}$ MCT per piglet led to greater duodenal and jejunal $\mathrm{VH}$ at $6 \mathrm{~h}$ and, 6 and 8 days of age. Also, the $\mathrm{VH}$ in the ileum at 8 days in pigs fed $5 \mathrm{~mL}$ MCT pet piglet was also significantly higher than that of the control. Thus, MCT not only enhanced nutrient absorption and utilization but also promoted the development of the intestines as a whole to improve growth performance. Consequently, the present study suggests that $0.1 \% \mathrm{MCT}$ given during phase 1 improved duodenal and ileal $\mathrm{VH}$ and strengthened the intestinal tracts, as shown in the previous studies of Czernichow et al [31] and 
Table 5. Effects of medium chain triglycerides and organic acids on blood profiles in weaning pigs ${ }^{1)}$

\begin{tabular}{|c|c|c|c|c|c|c|c|c|c|c|}
\hline \multirow{2}{*}{ Items } & \multicolumn{5}{|c|}{ Treatment $^{2)}$} & \multirow{2}{*}{ SEM } & \multicolumn{4}{|c|}{ p-value } \\
\hline & CON & LM & LMO & HM & HMO & & Diet & MCT & OA & MCT $\times O A$ \\
\hline \multicolumn{11}{|c|}{ Cortisol (ng/mL) } \\
\hline Initial & & 1621 & ---44.56 & 30 & 1014 & & & & & \\
\hline $2 w k$ & 5.48 & 16.24 & 4.68 & 25.36 & 10.14 & 3.551 & 0.15 & 0.29 & 0.06 & 0.78 \\
\hline 5 wk & 24.33 & 14.68 & 10.58 & 20.93 & 18.28 & 2.887 & 0.17 & 0.27 & 0.58 & 0.90 \\
\hline \multicolumn{11}{|c|}{$\operatorname{lgG}(\mathrm{g} / \mathrm{mL})$} \\
\hline Initial & - & 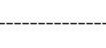 & -9.00 & -1----- & -1-2-1- & - & - & - & - & - \\
\hline $2 w k$ & 10.43 & 11.73 & 10.35 & 11.01 & 10.68 & 0.492 & 0.87 & 0.85 & 0.43 & 0.63 \\
\hline 5 wk & 15.67 & 19.04 & 14.14 & 15.15 & 17.39 & 1.113 & 0.31 & 0.88 & 0.56 & 0.13 \\
\hline \multicolumn{11}{|c|}{ TNF-a (pg/mL) } \\
\hline Initial & & & --127.89 & -------1 & 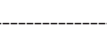 & & & & & \\
\hline $2 w k$ & 147.85 & 137.85 & 137.78 & 117.01 & 124.94 & 5.114 & 0.06 & 0.11 & 0.70 & 0.70 \\
\hline 5 wk & 134.94 & 118.18 & 130.34 & 106.13 & 110.33 & 5.792 & 0.42 & 0.19 & 0.49 & 0.73 \\
\hline \multicolumn{11}{|c|}{ IL-6 (pg/mL) } \\
\hline Initial & $\cdots$ & 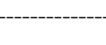 & ---317.56 & ------ & ----י- & & & & & \\
\hline $2 w k$ & 320.49 & 352.14 & 329.99 & 407.52 & 310.32 & 51.535 & 0.89 & 0.88 & 0.62 & 0.75 \\
\hline $5 w k$ & 311.86 & 281.42 & 289.32 & 293.08 & 288.34 & 34.498 & 0.99 & 0.94 & 0.98 & 0.93 \\
\hline \multicolumn{11}{|c|}{$\mathrm{IL}-10(\mathrm{pg} / \mathrm{mL})$} \\
\hline Initial & & -----י- & --- 7.94 & - & - & & & & & \\
\hline $2 w k$ & 7.33 & 6.48 & 9.95 & 7.62 & 6.17 & 0.661 & 0.07 & 0.30 & 0.42 & 0.06 \\
\hline $5 w k$ & 6.72 & 7.46 & 9.37 & 7.58 & 8.77 & 0.879 & 0.86 & 0.90 & 0.42 & 0.84 \\
\hline \multicolumn{11}{|c|}{$\mathrm{IL}-1 \beta(\mathrm{pg} / \mathrm{mL})$} \\
\hline Initial & & & $--23.61-$ & & & & & & & \\
\hline $2 w k$ & 18.29 & 17.56 & 27.47 & 28.59 & 24.54 & 2.928 & 0.15 & 0.52 & 0.64 & 0.28 \\
\hline $5 w k$ & 23.28 & 12.94 & 19.49 & 26.99 & 19.81 & 3.898 & 0.28 & 0.41 & 0.97 & 0.43 \\
\hline
\end{tabular}

SEM, standard error of the mean; MCT, medium chain triglyceride; OA, organic acids; ng, nanogram; pg, picogram; IgG, immunoglobulin G; TNF-a, tumor necrosis factor-a; IL, interleukin; SBM, soybean meal.

1) Least squares means of 5 observations per treatment.

2) CON, corn-SBM based diet; LM, corn-SBM based diet + MCT 0.1\%; LMO, corn-SBM based diet + MCT 0.1\% + organic acids $0.1 \%$; HM, corn-SBM based diet + MCT 0.2\%; HMO, corn-SBM based diet + MCT $0.2 \%$ + organic acids $0.1 \%$.

Yen et al [14]. Villi have finer-like protrusions in the epithelial inner layer that help to increase the surface area for digestion and absorption processes [32]. Since the improvement in $\mathrm{VH}$ increases absorption and surface area, it directly affects the ability of the intestine to absorb nutrients. Mekbungwan and Yamauchi [33] also found a highly positive relationship between an increased $\mathrm{VH}$ and improved growth performance of piglets.

Furthermore, the results of this current experiment showed that $\mathrm{VH}$ was increased by the $0.1 \% \mathrm{OA}$ supplementation during phase 2 and were similar to those of Long et al [34] and Ferrara et al [35]. According to the previous study of Long et al [34], supplementing $0.2 \%$ OA1 (a synergistic blend of free and buffered short chain fatty acids [mainly formic acid, acetic acid, and propionic acid]) combined with MCT tended to increase ileal $\mathrm{VH}(\mathrm{p}=0.07)$ in weaning pigs. Also, the ratio of $\mathrm{VH}: \mathrm{CD}$ for the jejunum and ileum was greater $(\mathrm{p}<0.05)$ for pigs fed OA1 and OA2 (a synergistic blend of a phenolic compound) compared to the control. Ferrara et al [35] have also shown that $\mathrm{VH}$ and $\mathrm{CD}$ in the jejunum were slightly higher in the treatment groups fed $1.05 \% \mathrm{OA}$ and
$1.05 \%$ OA with $0.3 \%$ MCT than in the control in weaning pigs. The authors reported that the supplementation of OA and MCT may result in decreased apoptosis rates at the top of the villi. Therefore, the increase in $\mathrm{VH}$ and $\mathrm{VH}: \mathrm{CD}$ ratio in the duodenum during phase 2 in this current experiment could be the result of $0.1 \%$ OA supplementation and effects increasing $\mathrm{VH}$ with regards to the results of Long et al [34] and Ferrara et al [35].

Therefore, the supplementation of MCT with OA, as in many previous studies, influenced the morphological changes of the small intestine, such as increased VH. In particular, $0.1 \%$ MCT significantly increased duodenal and ileal VH during phase 1 , and $0.1 \% \mathrm{OA}$ significantly increased $\mathrm{VH}$ and $\mathrm{VH}: \mathrm{CD}$ ratio in the duodenum during phase 2 in this current experiment. Overall, supplementation of MCT and OA could improve the absorption and utilization of nutrients to enhance the growth performance.

\section{Nutrient digestibility}

The effects of MCT with OA on nutrient digestibility and nitrogen retention are presented in Table 7. Adding MCT and 
Table 6. Effects of medium chain triglycerides and organic acids on histomorphology of small intestine in weaning pigs ${ }^{1)}$

\begin{tabular}{|c|c|c|c|c|c|c|c|c|c|c|}
\hline \multirow{2}{*}{ Items } & \multicolumn{5}{|c|}{ Treatment $^{2)}$} & \multirow{2}{*}{ SEM } & \multicolumn{4}{|c|}{ p-value } \\
\hline & CON & LM & LMO & HM & HMO & & Diet & MCT & $O A$ & MCT $\times O A$ \\
\hline \multicolumn{11}{|l|}{$2 w k$} \\
\hline Villus height ( $\mu \mathrm{m})$ & 312.67 & 340.26 & 341.08 & 291.83 & 251.87 & 14.687 & 0.33 & 0.04 & 0.51 & 0.49 \\
\hline Crypt depth $(\mu \mathrm{m})$ & 311.29 & 329.86 & 350.44 & 322.61 & 363.41 & 13.627 & 0.90 & 0.92 & 0.33 & 0.74 \\
\hline VH:CD & 1.00 & 1.03 & 0.97 & 0.90 & 0.69 & 0.062 & 0.37 & 0.13 & 0.29 & 0.47 \\
\hline Crypt depth ( $\mu m)$ & 235.07 & 236.29 & 245.73 & 237.33 & 274.68 & 14.016 & 0.84 & 0.64 & 0.47 & 0.66 \\
\hline $\mathrm{VH}: \mathrm{CD}$ & 1.17 & 1.33 & 1.33 & 1.18 & 1.13 & 0.085 & 0.95 & 0.36 & 0.97 & 0.99 \\
\hline \multicolumn{11}{|l|}{ Ileum } \\
\hline Villus height ( $\mu \mathrm{m})$ & 231.82 & 272.76 & 292.61 & 234.63 & 253.37 & 10.877 & 0.16 & 0.03 & 0.24 & 0.97 \\
\hline Crypt depth $(\mu \mathrm{m})$ & 231.23 & 245.27 & 261.61 & 223.23 & 257.69 & 13.962 & 0.76 & 0.61 & 0.34 & 0.72 \\
\hline Crypt depth ( $\mu \mathrm{m})$ & 386.08 & 397.83 & 327.80 & 417.09 & 376.71 & 22.609 & 0.70 & 0.49 & 0.27 & 0.76 \\
\hline $\mathrm{VH}: \mathrm{CD}$ & 0.70 & 0.61 & 0.95 & 0.65 & 0.78 & 0.071 & 0.11 & 0.43 & 0.04 & 0.17 \\
\hline \multicolumn{11}{|l|}{ Jejunum } \\
\hline Villus height ( $\mu \mathrm{m})$ & 229.19 & 234.02 & 281.25 & 242.76 & 277.82 & 11.252 & 0.41 & 0.91 & 0.14 & 0.81 \\
\hline Crypt depth ( $\mu \mathrm{m})$ & 291.93 & 298.49 & 250.48 & 242.08 & 266.93 & 11.035 & 0.60 & 0.36 & 0.59 & 0.11 \\
\hline $\mathrm{VH}: \mathrm{CD}$ & 0.78 & 0.78 & 1.12 & 1.00 & 1.04 & 0.056 & 0.25 & 0.45 & 0.10 & 0.24 \\
\hline \multicolumn{11}{|l|}{ Ileum } \\
\hline Villus height ( $\mu \mathrm{m})$ & 198.17 & 255.16 & 262.81 & 255.28 & 255.85 & 21.163 & 0.69 & 0.92 & 0.91 & 0.92 \\
\hline Crypt depth $(\mu \mathrm{m})$ & 215.74 & 242.83 & 246.05 & 242.16 & 230.89 & 15.841 & 0.97 & 0.83 & 0.91 & 0.84 \\
\hline $\mathrm{VH}: \mathrm{CD}$ & 0.91 & 1.05 & 1.06 & 1.05 & 1.10 & 0.092 & 0.96 & 0.94 & 0.82 & 0.77 \\
\hline
\end{tabular}

SEM, standard error of the mean; MCT, medium chain triglyceride; OA, organic acids; $\mathrm{VH}: \mathrm{CD}$, villus height to crypt depth ratio; SBM, soybean meal.

1) Least squares means for three pigs per treatment.

2) CON, corn-SBM based diet; LM, corn-SBM based diet + MCT 0.1\%; LMO, corn-SBM based diet + MCT 0.1\% + organic acids $0.1 \%$; HM, corn-SBM based diet + MCT 0.2\%; HMO, corn-SBM based diet + MCT $0.2 \%$ + organic acids $0.1 \%$.

OA did not affect nutrient digestibility and nitrogen retention.

However, unlike the present results, previous studies have shown that MCT supplementation improved nutrient di- gestibility. Han et al [29] have reported that compared to antibiotic treatment, supplementing $0.1 \%$ MCT significantly improved CP, calcium, phosphorus, and energy digestibility

Table 7. Effects of medium chain triglycerides and organic acids on nutrient digestibility in weaning pigs ${ }^{1)}$

\begin{tabular}{|c|c|c|c|c|c|c|c|c|c|c|}
\hline \multirow{2}{*}{ Items } & \multicolumn{5}{|c|}{ Treatment $^{2)}$} & \multirow{2}{*}{ SEM } & \multicolumn{4}{|c|}{$\mathrm{p}$-value } \\
\hline & CON & LM & LMO & HM & HMO & & Diet & MCT & $O A$ & MCT $\times O A$ \\
\hline \multicolumn{11}{|c|}{ Nutrient digestibility (\%) } \\
\hline Dry matter & 96.20 & 96.82 & 95.78 & 96.29 & 95.68 & 0.417 & 0.91 & 0.74 & 0.40 & 0.82 \\
\hline Crude protein & 95.70 & 96.39 & 94.57 & 95.91 & 95.06 & 0.538 & 0.77 & 0.99 & 0.25 & 0.66 \\
\hline Crude fat & 85.18 & 86.66 & 83.66 & 85.00 & 83.33 & 1.597 & 0.96 & 0.78 & 0.53 & 0.85 \\
\hline Crude ash & 90.32 & 92.00 & 89.00 & 90.00 & 89.67 & 1.071 & 0.93 & 0.78 & 0.50 & 0.59 \\
\hline \multicolumn{11}{|l|}{ N-retention $(\mathrm{g} / \mathrm{d})$} \\
\hline N-intake & 7.05 & 6.98 & 6.45 & 6.91 & 7.17 & 2.557 & - & - & - & - \\
\hline $\mathrm{N}$-feces & 2.14 & 1.76 & 2.63 & 1.96 & 2.54 & 0.258 & 0.76 & 0.92 & 0.21 & 0.79 \\
\hline N-urine & 3.03 & 2.52 & 3.78 & 2.83 & 3.54 & 0.362 & 0.74 & 0.96 & 0.23 & 0.72 \\
\hline N-retention ${ }^{3)}$ & 1.88 & 2.70 & 0.04 & 2.12 & 1.09 & 0.633 & 0.68 & 0.65 & 0.72 & 0.20 \\
\hline
\end{tabular}

SEM, standard error of the mean; MCT, medium chain triglyceride; OA, organic acids; SBM, soybean meal.

1) A total of 15 barrows (initial body weight, $12.48 \pm 0.37 \mathrm{~kg}$ ) were used.

2) CON, corn-SBM based diet; LM, corn-SBM based diet + MCT 0.1\%; LMO, corn-SBM based diet + MCT 0.1\% + organic acids $0.1 \%$; HM, corn-SBM based diet + MCT 0.2\%; HMO, corn-SBM based diet + MCT 0.2\% + organic acids $0.1 \%$.

${ }^{3)} \mathrm{N}$ retention $=\mathrm{N}$ intake $(\mathrm{g})-$ fecal $\mathrm{N}(\mathrm{g})-$ urinary $\mathrm{N}(\mathrm{g})$. 
in weaning pigs. Han et al [29] have also reported that lipids affected nutrient digestibility by changing the morphology of the small intestine. In addition, Li et al [23] have reported that supplementation of $1.4 \%$ MCT showed significantly higher digestibility of ether extract compared to the control in weaning pigs during day 12 to 14 and day 26 to 28 . This was because MCT improved the malabsorption of fat in the case of a contracted absorptive surface or atrophied intestinal villi. Moreover, the treatment supplemented with $1.4 \%$ MCT had a better result in the apparent total tract digestibility of DM, which resulted from an integrated improvement in the digestibility of various nutrients.

In conclusion, there are many previous studies where supplementation of MCT resulted in improved nutrient digestibility, but no significant difference was found in this study with MCT and OA additions. The discrepancy may be due to the differences in the composition of the diets, component ratio, and experimental situation. In the case of the Han's experiment, the experiment was conducted only in supplementation with antibiotics and $\mathrm{ZnO}$ not in the control in terms of digestibility. Also, the form of the diet (crumbled vs powdered form) was different which could possibly affect feed intake and digestibility. In the case of the Lis experiment, the amount of MCT supplemented was $0.7 \%, 1.4 \%$, and $2.1 \%$, which was different from the $0.1 \%$ and $0.2 \% \mathrm{MCT}$ added in this experiment. These differences could bring different results with this experiment.

\section{CONCLUSION}

The addition of $0.1 \%$ MCT in the diets for weaning pigs improved the growth performance of pigs with the increase of duodenal and ileal $\mathrm{VH}$. The addition of $0.1 \% \mathrm{OA}$ in the diets for weaning pigs increased duodenal $\mathrm{VH}$ and $\mathrm{VH}: \mathrm{CD}$. However, increasing the level of dietary MCT from $0.1 \%$ to $0.2 \%$ supplemented with $0.1 \%$ OA showed a negative effect on the growth performance of weaning pigs. Therefore, the inclusion of $0.1 \% \mathrm{MCT}$ and $0.1 \% \mathrm{OA}$ in the diets for weaning pigs would be beneficial for the growth performance of pigs with an improvement of the gut environment.

\section{CONFLICT OF INTEREST}

We certify that there is no conflict of interest with any financial organization regarding the material discussed in the manuscript.

\section{FUNDING}

The authors received no financial support for this article.

\section{ACKNOWLEDGMENTS}

This research was supported by E\&T Co., Ltd (Daejeon, Korea).

\section{REFERENCES}

1. Campbell JM, Crenshaw JD, Polo J. The biological stress of early weaned piglets. J Anim Sci Biotechnol 2013;4:19. https:// doi.org/10.1186/2049-1891-4-19

2. Stanton TB. A call for antibiotic alternatives research. Trends Microbiol 2013;21:111-3. https://doi.org/10.1016/j.tim.2012. 11.002

3. Kuang Y, Wang Y, Zhang Y, et al. Effects of dietary combinations of organic acids and medium chain fatty acids as a replacement of zinc oxide on growth, digestibility and immunity of weaned pigs. Anim Feed Sci Technol 2015;208: 145-57. https://doi.org/10.1016/j.anifeedsci.2015.07.010

4. Odle J. New insights into the utilization of medium-chain triglycerides by the neonate: observations from a piglet model. J Nutr 1997;127:1061-7. https://doi.org/10.1093/jn/127.6.1061

5. Dierick NA, Decuypere JA, Degeyter I. The combined use of whole cuphea seeds containing medium chain fatty acids and an exogenous lipase in piglet nutrition. Arch Anim Nutr 2003;57:49-63. https://doi.org/10.1080/0003942031000086626

6. Bergsson G, Arnfinnsson J, Steingrimsson O, Thormar H. Killing of Gram-positive cocci by fatty acids and monoglycerides. APMIS 2001;109:670-8. https://doi.org/10.1034/j. 1600-0463.2001.d01-131.x

7. Sk`rivanová E, Marounek M, Benda V, Brezina P. Susceptibility of Escherichia coli, Salmonella sp and Clostridium perfringens to organic acids and monolaurin. Vet Med Czech 2006;51: 81-8.

8. Lawlor PG, Lynch PB, Caffrey PJ. Effect of fumaric acid, calcium formate and mineral levels in diets on the intake and growth performance of newly weaned pigs. Irish J Agric Food Res 2006;45:61-71.

9. Partanen K, Mroz Z. Organic acids for performance enhancement in pig diets. Nutr Res Rev 1999;12:117-45. https://doi. org/10.1079/095442299108728884

10. Lei XJ, Park JW, Baek DH, Kim JK, Kim IH. Feeding the blend of organic acids and medium chain fatty acids reduces the diarrhea in piglets orally challenged with enterotoxigenic Escherichia coli K88. Anim Feed Sci Technol 2017;224:4651. https://doi.org/10.1016/j.anifeedsci.2016.11.016

11. Kim BG, Lindemann MD. A spreadsheet method for the experimental animal allotment. J Anim Sci 2007;85:112.

12. NRC. Nutrient requirements of swine. 10th Ed. Washington, DC, USA: National Academy Press; 1998.

13. NRC. Nutrient requirements of swine. 11th Ed. Washington, DC, USA: National Academy Press; 2012.

14. Yen HC, Lai WK, Lin CS, Chiang SH. Medium-chain trigly- 
ceride as an alternative of in-feed colistin sulfate to improve growth performance and intestinal microbial environment in newly weaned pigs. Anim Sci J 2015;86:99-104. https:// doi.org/10.1111/asj.12248

15. McCarthy JF, Aherne FX, Okai DB. Use of HCL insoluble ash as an index material for determining apparent digestibility with pigs. Can J Anim Sci 1974;54:107-9. https://doi.org/10. 4141/cjas74-016

16. Kim BG, Lee JW, Stein HH. Energy concentration and phosphorus digestibility in whey powder, whey permeate, and low-ash whey permeate fed to weanling pigs. J Anim Sci 2012; 90:289-95. https://doi.org/10.2527/jas.2011-4145

17. AOAC. Official methods of analysis. 16th Edition. Washington, DC, USA: Association of Official Analytical Chemists; 1995.

18. Loh TC, Foo HL, Thanh NT, Choe DW. Growth performance, plasma fatty acids, villous height and crypt depth of preweaning piglets fed with medium chain triacylglycerol. AsianAustralas J Anim Sci 2013;26:700-4. https://doi.org/10.5713/ ajas.2012.12561

19. Dierick NA, Decuypere JA, Molly K, Vanbeek E, Vanderbecke E. The combined use of triacylglycerols (TAGs) containing medium chain fatty acids (MCFAs) and exogenous lipolytic enzymes as an alternative to nutritional antibiotics in piglet nutrition: II. In vivo release of MCFAs in gastric cannulated and slaughtered piglets by endogenous and exogenous lipases; effects on the luminal gut flora and growth performance. Livest Prod Sci 2002;76:1-16. https://doi.org/10.1016/S03016226(01)00331-1

20. Sha ND, Limketkai BN. The use of medium chain triglycerides in gastrointestinal disorders. Practi Gastroenterol 2017;160: 20-8.

21. Limketkai BN, Zucker SD. Hyperammonemic encephalopathy caused by carnitine deficiency. J Gen Intern Med 2008; 23:210-3. https://doi.org/10.1007/s11606-007-0473-0

22. Allee GL, Romsos DR, Leveille GA, Baker DH. Metabolic consequences of dietary medium-chain triglycerides in the pig. Proc Soc Exp Biol Med 1972;139:422-8. https://doi.org/ 10.3181/00379727-139-36158

23. Li Y, Zhang H, Li Y, Zhang L, Wang T. Effect of medium-chain triglycerides on growth performance, nutrient digestibility, plasma metabolites and antioxidant capacity in weanling pigs. Anim Nutr 2015;1:12-8. https://doi.org/10.1016/j.aninu. 2015.02.001

24. Tee LH, Ahmed EAE, Toung OP, Hong OC. Effect of mediumchain triglycerides on piglets in three farms in Selangor and Penang, Malaysia. In: 5th Proceedings of the Seminar in Veterinary Sciences; 2010 Jan 5-8. Serdang, Malaysia: Universiti
Putra Malaysia Press; 2010.

25. Petschow BW, Batema RP, Talbott RD, Ford LL. Impact of medium-chain monoglycerides on intestinal colonization by Vibrio 70 cholera or enterotoxigenic Escherichia coli. J Med Microbiol 1998;47:383-9.

26. Tsiloyiannis VK, Kyriakis SC, Vlemmas J, Sarris K. The effects of organic acids on the control of porcine post-weaning diarrhea. Vet Sci Res J 2001;70:287-93. https://doi.org/10. 1053/rvsc.2001.0476

27. Yang Y, Lee KY, KIM IH. Effects of dietary protected organic acids on growth performance, nutrient digestibility, fecal microflora, diarrhea score, and fecal gas emission in weanling pigs. Can J Anim Sci 2019;99:514-20. https://doi.org/10.1139/ cjas-2018-0159

28. Cook NJ, Schaefer AL, Lepage P, Jones SM. Salivary vs. serum cortisol for the assessment of adrenal activity in swine. Can J Anim Sci 1996;76:329-35. https://doi.org/10.4141/cjas96049

29. Han YK, Hwang IL, Thacker PA. Use of a micro-encapsulated eucalyptus-medium chain fatty acid product as an alternative to zinc oxide and antibiotics for weaned pigs. J Swine Health Prod 2011;19:34-43.

30. Kelley KW, Johnson RW, Dantzer R. Immunology discovers physiology. Vet Immunol Immunopathol 1994;43:157-65. https://doi.org/10.1016/0165-2427(94)90132-5

31. Czernichow B, Galluser M, Cui SQ, Gosse F, Raul F. Comparison of enteral or parenteral administration of medium chain triglycerides on intestinal mucosa in adult rats. Nutr Res 1996;16:797-804. https://doi.org/10.1016/0271-5317(96) 00072-3

32. Fuller MF. The encyclopedia of farm animal nutrition. UK: CAB International Publishing; 2004.

33. Mekbungwan A, Yamauchi K. Growth performance and histological intestinal alterations in piglets fed dietary raw and heated pigeon pea seed meal. Histol Histopathol 2004; 19:381-9. https://doi.org/10.14670/HH-19.381

34. Long SF, Xu YT, Pan L, et al. Mixed organic acids as antibiotic substitutes improve performance, serum immunity, intestinal morphology and microbiota for weaned piglets. Anim Feed Sci Technol 2018;235:23-32. https://doi.org/10.1016/j.ani feedsci.2017.08.018

35. Ferrara F, Tedin L, Pieper R, Meyer W, Zentek J. Influence of medium-chain fatty acids and short-chain organic acids on jejunal morphology and intra-epithelial immune cells in weaned piglets. J Anim Phys Anim Nutr 2017;101:531-40. https://doi.org/10.1111/jpn.12490 